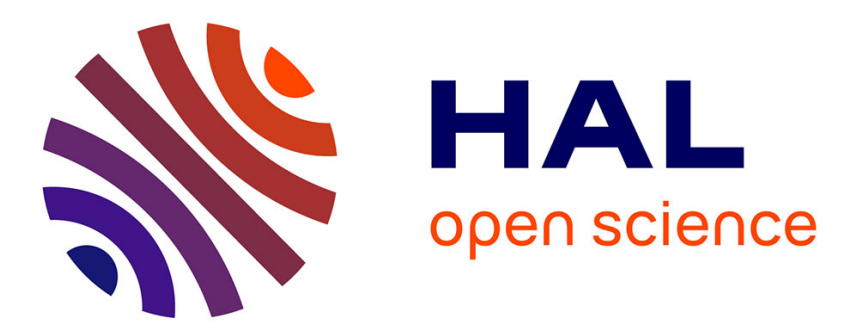

\title{
2-and-3D Analysis of temperature effects on periodic transducers using a FEA/BEM approach
}

J. Garcia, Thierry Laroche, W. Daniau, E. Carry, G. Martin, S. Ballandras, J.-M. Friedt

\section{To cite this version:}

J. Garcia, Thierry Laroche, W. Daniau, E. Carry, G. Martin, et al.. 2-and-3D Analysis of temperature effects on periodic transducers using a FEA/BEM approach. Joint Meeting of the 23rd European Frequency and Time Forum/IEEE International Frequency Control Symposium, Apr 2009, Besançon, France. pp.424-429, 10.1109/FREQ.2009.5168214 . hal-00533739

\section{HAL Id: hal-00533739 \\ https://hal.science/hal-00533739}

Submitted on 24 Mar 2021

HAL is a multi-disciplinary open access archive for the deposit and dissemination of scientific research documents, whether they are published or not. The documents may come from teaching and research institutions in France or abroad, or from public or private research centers.
L'archive ouverte pluridisciplinaire HAL, est destinée au dépôt et à la diffusion de documents scientifiques de niveau recherche, publiés ou non, émanant des établissements d'enseignement et de recherche français ou étrangers, des laboratoires publics ou privés. 


\section{2-and-3D Analysis of temperature effects on periodic transducers using a FEA/BEM approach}

\author{
Julien Garcia, Thierry Laroche, William Daniau, Emile \\ Carry, Gilles Martin, S. Ballandras \\ FEMTO-ST, UMR 6174 CNRS-UFC-ENSMM-UTBM \\ Besançon, France \\ sylvain.ballandras@femto-st.fr
}

\author{
Jean-Michel Friedt \\ SENSeOR SAS \\ Sophia Antipolis, France \\ jmfriedt@femto-st.fr
}

\begin{abstract}
The thermal sensitivity of acoustic-wave-based devices still is an optimization key-point for various applications (filters, sources, sensors). As the architecture of such devices becomes more and more complex to fit the RF manufacturer requirements, simple analytical models usually exploited to optimize their frequency thermal drift are found obsolete. The optimization of these complex periodic wave-guide thermal sensitivities require the development of simulation tools capable to address the problem. This paper describes a Finite Element Analysis-Boundary Element Method (FEA/BEM) model allowing for the simulation any $2 \mathrm{D}$ and $3 \mathrm{D}$ periodic wave-guide thermal sensitivity, and more specifically the extraction of the corresponding Temperature Coefficient of Frequency (TCF). Validation of the computation approach first is reported. Its capabilities then are illustrated by computing the TCF of Love wave resonator on quartz.
\end{abstract}

\section{INTRODUCTION}

The control of thermal stability of acoustic wave transducers still is one of the major problems in developing advanced solutions for passive radio-frequency (RF) signal processing. Controlling the thermal frequency drift of such devices enables one to reduce the transition bandwidth of such filters, then increasing their selectivity and allowing for high density spectrum channeling. Different approaches have been tested to efficiently address this problem, yielding complex transducer architectures combining highly coupled single crystal substrates with various overlays. Also new kinds of wave excitation and guiding have been imagined and tested in that purpose, such as interface waves. The same kind of challenge occurs for film bulk acoustic resonators (FBARs), the very serious alternative to SAW when addressing telecommunication standard frequency rising for instance, for which a combination of positive and negative thermal drift material allows for reducing the overall temperature sensitivity of the final devices.

We have been developing a mixed finite element/boundary element modeling code capable to simulate almost any kind of 1D-periodic as well as 2D-periodic transducer structures, accounting for acoustic radiation above and below the waveguide and of course different nature of material losses (viscoelastic, dielectric). In this work, this code has been enhanced to account for the thermal evolution of material characteristics as well as wave-guide thermal expansion to simulate the temperature dependence of guided (or partially guided or even radiated) modes in layered transducer structures. The basis of these developments consists of a material coefficient perturbation according to the celebrated Campbell \& Jones approach [1], with an appropriate strategy to expanse the meshed region along temperature. In these developments, we neglect thermo-differential stresses. This represents a severe limitation of this approach, but reveals reasonable for achieving reliable predictions of the actual SAW Temperature Coefficients of Frequency (TCF) as demonstrated by Pastureaud \& al [2]. The principle of the calculation then consists in computing the harmonic admittance of a given transducer at different temperatures and to automatically extract parameters such as resonance and antiresonance frequencies (and also the stop-band width when accessible). The thermal sensitivity of these parameters then is obtained by fitting the results considering quadratic or cubic temperature dependence according to the regarded configuration.

In this paper, we expose the basic theory of our temperaturedependent model, and we show various results validating the proposed approach for bulk acoustic wave (BAW) and SAW devices. We then propose to simulate more complex transducer structures such as thick guiding layers, or even interface wave transducer, to illustrate the capability of the model to address complex guiding structures without restriction. 


\section{TheORETICAL BASIS}

\section{A. FEA-BEM Model}

As already reported in many references (the most detailed description of the model will be found in ref. [3]), FEA can be performed for periodic devices with rather simple modifications of the basic algebraic formula associating the displacement and electrical fields to the boundary solicitations. Figure 1 shows the typical configuration for which the model was developed. We assume an inhomogeneous region of the wave-guide treated by finite element techniques above semi-infinite or finite thickness homogeneous regions (the substrate) treated by boundary integral methods. Note that the substrate can be composed of a single material or a stack of layers provided flat interfaces between the involved materials.

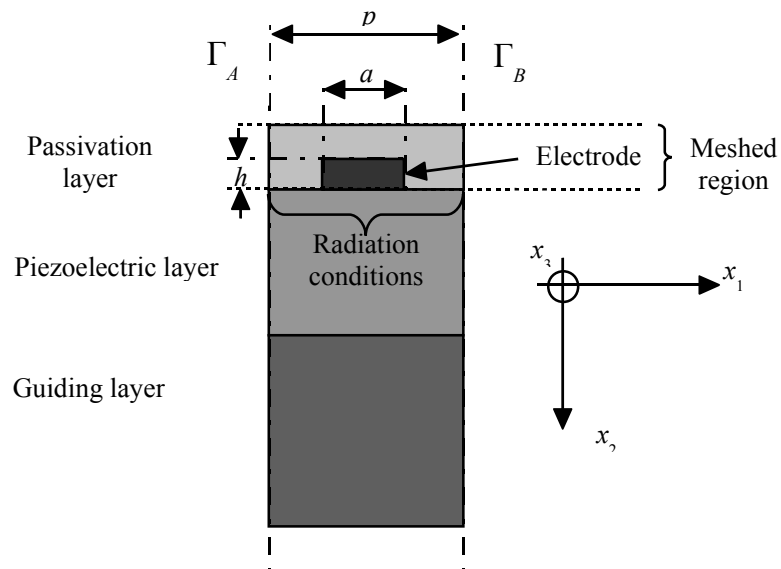

Figure 1. Typical 1-D periodic 2D wave-guide structure addressed by the model

Considering the harmonic excitation illustrated by (1), we relate all the degrees of freedom (dof) on boundary $\Gamma_{A}$ to those on boundary $\Gamma_{B}$, yielding the following expression

$$
\left\{\begin{array}{l}
u_{\Gamma_{B}} \\
\varphi_{\Gamma_{B}}
\end{array}\right\}=\left\{\begin{array}{l}
u_{\Gamma_{A}} \\
\varphi_{\Gamma_{A}}
\end{array}\right\} e^{-j 2 \pi \gamma}
$$

in which $u$ represents the mechanical displacements and $\phi$ the electrical potential. The excitation parameter $\gamma$ allows to account for the harmonic excitation figure applied to the array (see ref. [4] for a comprehensive definition of this parameter). This relation then is used to reduce the number of independent dof of the FEA model. This is performed without changing the total number of dof of the problem and by simply using a variable change operator $C$ depending on the excitation parameter $\gamma$. This provides the following form of the FEA algebraic system to solve

$$
\begin{aligned}
& { }_{t}\left[\begin{array}{cc}
C_{u}(\gamma) & 0 \\
0 & C_{\varphi}(\gamma)
\end{array}\right]\left[\begin{array}{cc}
K_{u u}-\omega^{2} M_{u u} K_{u \varphi} \\
K_{\varphi u} & K_{\varphi \varphi}
\end{array}\right]\left[\begin{array}{cc}
C_{u}(\gamma) & 0 \\
0 & C_{\varphi}(\gamma)
\end{array}\right]\left\{\begin{array}{l}
v \\
\varphi
\end{array}\right)= \\
& { }^{[}\left[\begin{array}{cc}
C_{u}(\gamma) & 0 \\
0 & C_{\varphi}(\gamma)
\end{array}\right]\left\{\begin{array}{l}
F \\
Q
\end{array}\right\}
\end{aligned}
$$

where $K$ and $M$ are respectively the FEA stiffness and mass matrices, $v$ and $\varphi$ the independent dof of the problem and $F$ and $Q$ the right hand side boundary forces and electrical charges. The upper script $*$ denotes a complex conjugation. Since $K$ can be complex, the left hand side matrix in (2) is complex general (hermitian if $K \in \mathfrak{R}$ ), but sparse. These properties are considered when solving the problem. The algebraic system (2) is solved, allowing for the computation of the total electrical charge under active electrodes simply by nodal value summation. The harmonic (frequency and $\gamma$ dependent) admittance then is equal to the electrode current for a unit voltage excitation $\left(\varphi_{0}=1 \mathrm{~V}\right.$.).

We now regard the case of acoustic radiation on one border of the meshed domain. In that purpose, the general variationnal equation is considered, limited to the purely elastic problem without any loss of generality

$$
\iiint_{\Omega}\left(\rho \omega^{2} u_{i} \delta u_{i}-\frac{\partial \delta u_{i}}{\partial x_{j}} C_{i j k l} \frac{\partial u_{l}}{\partial x_{k}}\right) d V=\iint_{\Gamma} \delta u_{i} T_{i j} n_{j} d S
$$

in which $\delta u_{i}$ is the variationnal unknown and $n_{j}$ the normal to the boundary $\Gamma$ (bordering the domain $\Omega$ ) on which the radiation boundary condition may partially occur. The mass density is represented by $\rho$ and $C_{i j k l}$ holds for the elastic constants. Equation (3) is written in 3D but of course its restriction to $2 \mathrm{D}$ problems does not induce any fundamental difficulty. The right hand side of (3) then is considered separately. In this matter, one can relate the dynamic stress $T_{i j}$ to the displacement $u_{k}$ in the spectral domain (denoted by $\sim$ ) by using a Green's-function-based relation which generalizes the usual surface stress relation widely used in SAW modeling as follows

$$
\tilde{T}_{i j}=\tilde{G}_{i j k}\left(s_{1}, \omega\right) \tilde{u}_{k}
$$

Note that in this equation, the Green's function asymptotically behaves like $s_{1}$ when $s_{1}$ tends to infinity and this property must be carefully accounted for. Equation (4) allows one for considering any flat boundary for the application of the radiation conditions, even if tilted in the $\left(x_{1}, x_{2}\right)$ plane. Using the now well-established periodic Green's function formalism, the right hand side of (4) is expressed as

$$
\begin{gathered}
\iint_{\Gamma} \delta u_{i} T_{i j} n_{j} d S= \\
\iint_{\Gamma} \delta u_{i}(x) \sum_{l=-\infty}^{+\infty} G_{i j k}(\gamma+l, \omega) \frac{n_{j}}{p} e^{-j \frac{\gamma \pi}{p}(\gamma+l)\left(x-x^{\prime}\right)} u_{k}\left(x^{\prime}\right) d x^{\prime} d x
\end{gathered}
$$


with $p$ the period of the problem and $l$ the number of the current space harmonic. The classical FEA interpolation procedure then is applied to (5), yielding the final expression of the boundary radiation operator [3].

The contribution of the total radiating boundary to the global algebraic system to be solved then consists in a frequency and excitation parameter dependent matrix $X(\omega, \gamma)$ related to both dof and variationnal unknowns and consequently computed in the left hand side of (2). Even for materials without any losses, the final algebraic system is general complex without any specific mathematical characteristics. Also the sparse nature of the algebraic system is degraded due to the connection of all the dof one to the other via the radiation coupling.

\section{B. Thermal perturbation}

Temperature effects can be simulated rather efficiently along the so-called Campbell \& Jones approach [1]. It consists in computing the effective constants of materials once temperature has changed, along a Taylor development. Physical properties of the structure (elasticity, piezoelectricity, dielectric permittivity...) actually change with temperature, and the structure expand in a complicated manner as different materials may be involved. Such phenomena perturb the acousto-electric behavior of all the elements of the structure (the substrate and the layers which compose the wave-guide) and consequently yields a thermal drift of the synchronism frequency of the device. This frequency variation can be expressed as a relative function consisting in two major contributions in the vicinity of the reference temperature $\mathrm{T}_{0}=25^{\circ} \mathrm{C}$. The relative phase velocity variation which reads

$$
\frac{d V}{V}(T)=\frac{V(T)-V\left(T_{0}\right)}{V\left(T_{0}\right)}
$$

The relative propagation distance variation is written as follows

$$
\frac{d e}{e}(T)
$$

As the frequency is given as a first approximation by the relation $f=V / 2 e$ for BAW (with $e$ the actual thickness of the bulk resonator) and $f=V / \lambda$ for SAW (with $\lambda=2 p$, i.e. twice the mechanical period), the relative temperature sensitivity of the frequency reads

$$
\frac{d f}{f}(T)=\frac{d V}{V}(T)-\frac{d e}{e}(T)
$$

where $\lambda$ replaces e fort SAW. For each considered temperature poin, the electromechanical problem is solved simply by changing the effective physical constants (elastic, piezoelectric and dielectric) and the mass density composing the structure. In this matter, one has to compute the thermal sensitivity coefficients of all the physical constants involved in the model in all the space directions as follows

$$
\begin{gathered}
c_{i j k l}(T)=c_{i j k l}(T .) \sum_{n=0}^{N} \frac{1}{c_{i j k l}\left(T_{0}\right)} \frac{\partial^{(n)} c_{i j k l}}{\partial T}\left(T-T_{0}\right)^{n}=\sum_{n=0}^{N} \kappa_{i j k l}^{(n)} \Delta T^{n} \\
L_{x_{i}}(T)=\sum_{n=0}^{N} \frac{\partial^{(n)} L_{x_{i}}}{\partial T}\left(T-T_{0}\right)^{n}=\sum_{n=0}^{N} \eta_{x_{i}}^{(n)} \Delta T^{n}
\end{gathered}
$$

where the sensitivity coefficients $\kappa^{(n)}{ }_{i j k l}$ and the thermal expansion coefficient $\eta^{(n)}$ are experimentally determined and accessible in the literature up to the third order at least for Quartz. For most materials however, only first or at best second order coefficients have been measured. Concerning the mass density, it is related to the thermal expansion of the elementary crystal lattice $V_{m e}(T)$ as follows:

$$
\rho(T)=\frac{\rho\left(T_{0}\right)}{V_{o l}(T)} \quad \text { with } \quad V_{m e}(T)=L_{x_{l}} L_{x_{2}} L_{x_{3}}
$$

However, in the case of FEA-BEM computation, the FEA part must be expanded to actually account for the period or the layer thickness changes corresponding to (7). In the case of wave-guide composed of different materials (as for SAW with Aluminum electrodes atop thick single crystal substrates), a rigorous analysis of the problem needs a finite element analysis of the structure expansion accounting for differential thermal stresses. However, as in ref. [2], we assume here that the substrate imposes its expansion to any film deposited atop its surface, but these materials do expand in the direction normal to the propagation surface along their own physical properties. This assumption is confirmed by experimental observations [5] on thin epitaxial PZT layers and was found efficient in [2] to accurately simulate actual devices.

Following this process, the resonance frequency of any mode of the infinite grating or of the BAW resonator is computed for each temperature point to derive an analytical frequencytemperature law. We generally assume that this law can be established according to the numerous results obtained for BAW and SAW devices as a third order polynomial function which reads

$$
\frac{\Delta f}{f}={ }^{\theta} \alpha\left(T-T_{0}\right)+{ }^{\theta} \beta\left(T-T_{0}\right)^{2}+{ }^{\theta} \gamma\left(T-T_{0}\right)^{3}
$$

where ${ }^{\theta} \alpha,{ }^{\theta} \beta$ and ${ }^{\theta} \gamma$ respectively represent the first, second and third order TCF computed at room temperature $T_{0}\left(25^{\circ} \mathrm{C}\right)$. This computation process only has a sense if it allows for 
accurately identifying the resonance frequency at each considered temperature. In that purpose, we use a parametric isomorphic function describing the admittance close to the resonance. The parameters of the model then are computed using a 3-point fit method described in [6]. A simple approach for fitting the resonance frequency consists in fixing $\gamma$ to a value corresponding to the actual excitation conditions applied to the grating (typically $1 / 2$ for SAW) and then fit the admittance changes versus frequency only. The resonance then no more depend on the excitation parameter, yielding the following isomorphic fitting function

$$
Y(\omega)=Y_{r}(\omega)+\frac{Y_{p}(\omega)}{\omega_{p}-\omega}
$$

where $\omega_{p}$ represents the resonance frequency, and $Y_{p}(\omega)$ and $Y_{r}(\omega)$ hold for the pole magnitude and the other contributions respectively. The 3-point fitting method quickly converges and reveals stable for well-posed problems. It can also be applied to identify anti-resonance parameters, simply changing the admittance to impedance in (13). This approach holds faor SAW and BAW as well. Finally it must be noted that all these developments are valid also for wave guide simulated by periodic FEA only, in 2D and 3D as well. The method finally can be extended for any FEA structure submitted to quasi-static thermal perturbation provided the assumption of negligible thermo-differential effects remains valid.

\section{VALIDATION RESULTS}

\section{A. BAW on Quartz}

The very first case to treat in order to check the validity of such a calculation corresponds to BAW in quartz, for which second order compensation effects on AT cut is well known and predicted by simple analytical models. In that case, we consider a very simple mesh corresponding to a $2 \mathrm{D}$ plane surface resonator. The thickness was arbitrarily fixed to 50 $\mu \mathrm{m}$ to provide a resonance frequency near $35 \mathrm{MHz}$ considering AT quartz. The width of the resonator also was arbitrarily fixed, but this figure had no impact on the results as the electrodes extend along the whole surfaces, Setting $\gamma$ to an integer value (arbitrarily 1) yields the simulation of an infinitely long transducer, as it is generally the case for simple analytical models. For achieving the computation, Slobodnik et al. elastic, piezoelectric dielectric constants and density have been used [7] but the considered effective thermal coefficients of those physical constants were those of Bechmann et al. [8]. Figure 2 shows the implemented mesh and fig. $3 \mathrm{a} \& \mathrm{~b}$ show the evolution of the resonance frequency for pure shear BAW on AT and BT quartz cuts respectively. The comparison with analytical model results reveal a good agreement between the two approaches, yielding a first validation element. Only FEA was used ion this first validation step.

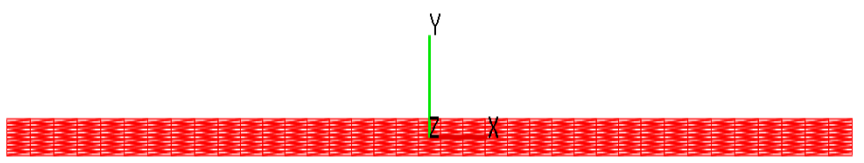

Figure 2. Mesh of the BAW resonator

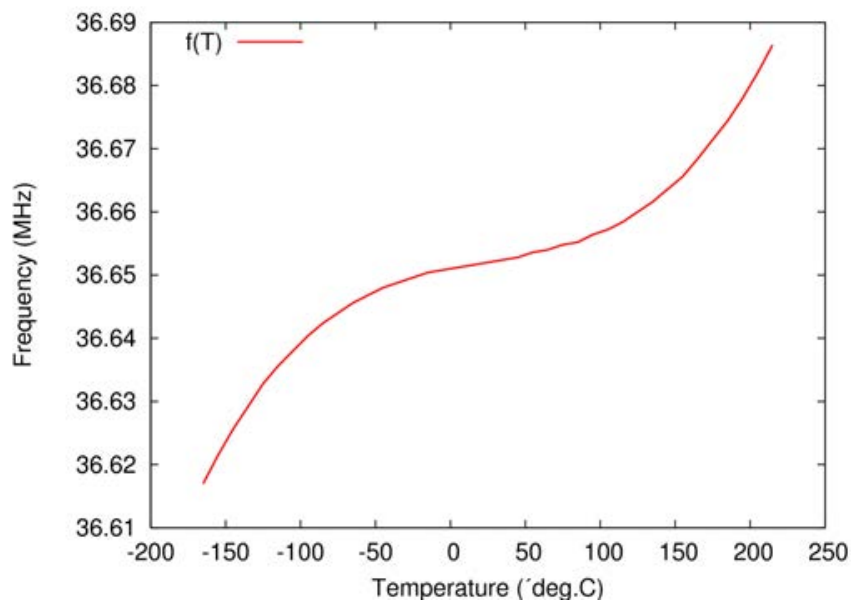

(a)

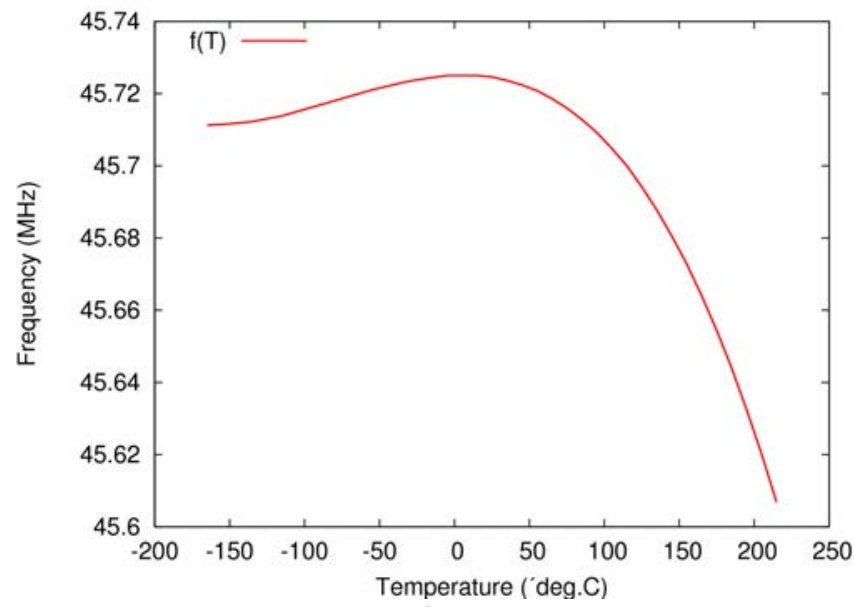

(b)

Figure 3. Thermal sensitivity of pure shear wave on (a) AT and (b) BT Quartz cuts computed along the proposed approach

\section{B. Rayleigh wave on Quartz}

The second unavoidable case to be addressed by the model corresponds to Rayleigh waves on (ST,X) Quartz and the sensitivity of the temperature compensation to electrode loading, as it also consists in a so-called "classics" which was treated along various approaches. In that case, we used the same data set as in section III.A, adding the contribution to Aluminum for which elastic and temperature coefficient was found in [9]. In that case, we combined FEA and BEM, both 
being thermally perturbed as described above. Figure 4 shows the implemented mesh and fig.5 shows the evolution of the Rayleigh wave frequency for 2 different metal thickness (100 and $200 \mathrm{~nm}$ ), the wavelength being arbitrarily set to $10 \mu \mathrm{m}$. It clearly shows that the turnover temperature shifts down with the electrode thickness, which actually corresponds to experimentally observed phenomena and agree well with theoretical results reported in [2].

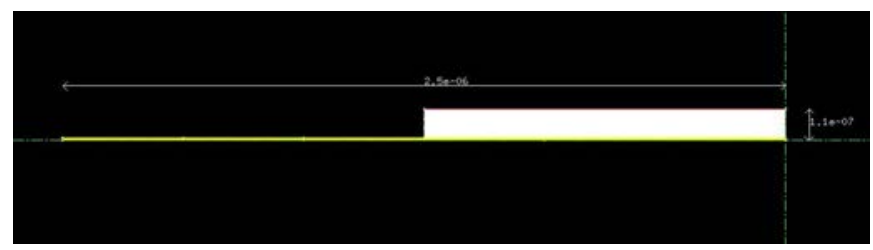

Figure 4. Mesh of the SAW grating, the metal ratio is set to 0.5 , the mechanical period equals $5 \mu \mathrm{m}$ and the electrode thickness varies from 100 to $200 \mathrm{~nm}$.

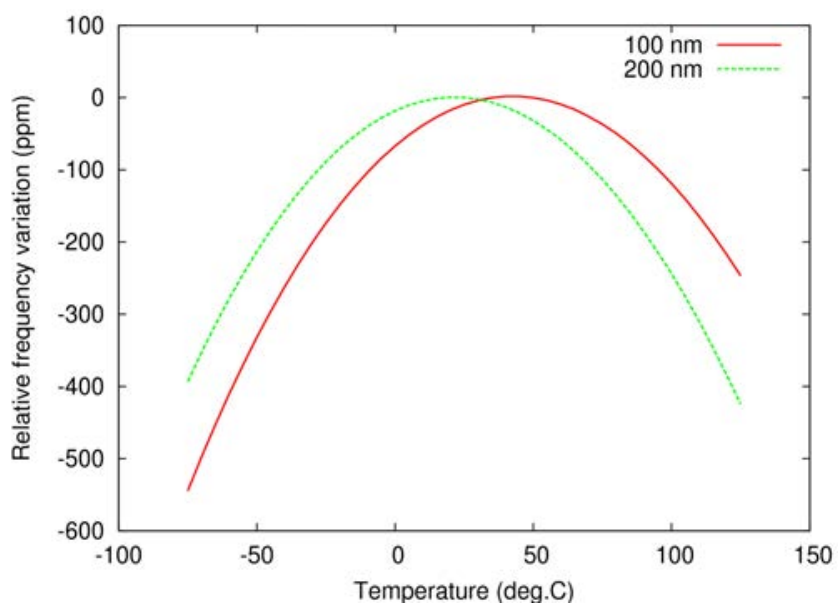

Figure 5. Thermal sensitivity of SAW under the above grating (fig.4) for 2 different electrode thickness (100 and $200 \mathrm{~nm}$ ).

\section{IV.THERMAL SENSITIVITY OF COMPLEX WAVE-GUIDE}

Passivation layers are used to protect the surface of SAW devices from any dusts or surface pollution that could counteract the IDT operation. To illustrate the capability of our model to correctly predict the response of complex surface structures and their particular thermal sensitivity, we have theoretically and experimentally check the influence of an amorphous silica $\left(\mathrm{SiO}_{2}\right)$ coating on a two port surface transverse wave (STW) resonator, yielding a so-called Love wave resonator. These devices consists in a four-port STW synchronous resonator operating close to $500 \mathrm{MHz}$ (grating period $p=5 \mu \mathrm{m}$, relative electrode height $h / \lambda=1.5 \%$, metallization ratio $a / p=0.5$ ) built on AT cut of quartz (propagation along $Z^{\prime}$ ) covered by a $0.5 \mu \mathrm{m}$ thick amorphous silica layer. It presents 2 resonances corresponding respectively to the beginning and the end of the frequency stop-band. The stop-band width only can be accurately predict if considering a conformal deposition, i.e. the corrugation due to the metal strip is reproduced at the silica surface, as shown in fig.6. A comparison between the harmonic admittance and the transfer function of the abovementioned resonator is reported in fig.7, showing a good agreement between theory and experiment. We then have applied our TCF calculation process, allowing for identifying the behavior of each independently. The results are reported in fig.8, showing that the two resonance does not exhibit exactly the same temperature behavior, although their polarization is about the same (phase quadrature). This actually corresponds to what is experimentally observed, even if the actual turnover temperatures occur at smaller temperature (cf Fig.9). This of course can be controlled by changing the effective elastic temperature constants, as they are not known as accurately as those of quartz.

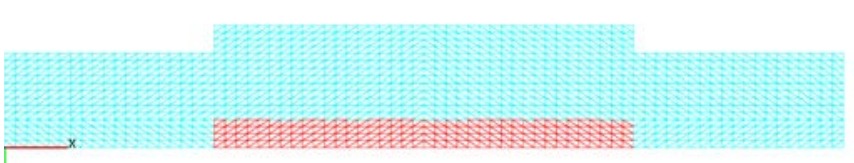

Figure 6. Mesh of the Love-wave grating, the metal ratio is set to 0.5 , the mechanical period equals $5 \mu \mathrm{m}$, the electrode thickness is set to $150 \mathrm{~nm}$ and the silica overlay is $0,5 \mu \mathrm{m}$ thick.

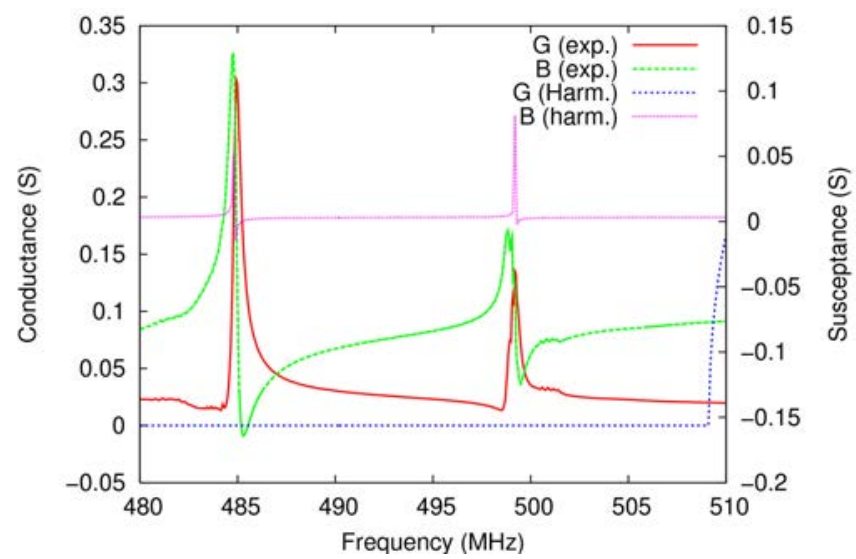

Figure 7. Superposition of harmonic admittances computed using the present approach and the above with the experimentally measured admittance of a passivated 4-port STW resonator

\section{CONCLUSION}

We have proposed an approach based on finite element analysis to simulate the thermal effects on the resonance frequency of complex wave guide. The basic computation has been validated for standard plane BAW resonators on Quartz. The possibility to integrate radiation effects using boundary elements based on Green's functions also has been validated for Rayleigh wave on quartz too, as the most difficult problem generally consists in accurately predicting the 
temperature compensation effects. This work is applied also to address Love wave device thermal behavior for which no simple model is capable to account for the influence of the IDT covered by th silica overlay. This work will be extended in the near future to address the problem of FBAR and SMR also requiring an accurate computation of the unperturbed model to correctly simulate the resonance conditions.

\section{REFERENCES}

[1] J.J. Campbell, W.R. Jones, "A method for estimating crystals cuts and propagation direction for excitation of piezoelectric surface waves", IEEE Trans. On Sonics and Ultrasonics, Vol. 15, pp. 209-217, 1968Ballandras \& al

[2] T. Pastureaud, R Lardat, S. Chamaly, L. Penavaire, S. Ballandras, Prediction of the thermal sensitivity of surface acoustic waves excited under a periodic grating of electrodes, Ultrasonics, Ferroelectrics and Frequency Control, IEEE Transactions on Volume 52, Issue 8, Aug. 2005 Page(s): $1378-1383$

[3] S. Ballandras, M. Wilm, P.F. Edoa, A. Soufyane, V. Laude,W. Steichen, R. Lardat, Finite-element analysis of periodic piezoelectric transducers,J. Appl. Phys. 93, 702 (2003); DOI:10.1063/1.1524711

[4] S. Ballandras, R. Lardat, M. Wilm, Th. Pastureaud, A. Reinhardt, N. Champavert, W. Steichen, W. Daniau, V. Laude, R. Armati, G. Martin, "A mixed finite element/boundary element approach to simulate complex guided elastic wave periodic transducers", Journal of Applied Physics, Volume 105, Issue 1, pp. 014911-014911-10 (2009)

[5] C. Lichtensteiger, M. Dawber and J.-M. Triscone, Ferroelectric size effects, Topics in applied physics 105, 305-338 (2007).

[6] Y. Fusero, S. Ballandras, J. Desbois, J.M. Hodé, P. Ventura, "SSBWto-PSAW conversion in SAW devices using heavy mechanical loading", IEEE Trans. on UFFC, Vol. 49, nº, pp. 805-814, 2002

[7] A.J Slobodnik, E.D Conway, R.T Delmonico , "Microwave Acoustics Handbook, vol 1A : Surface wave velocities", Air force Cambridge Research Labs, 1973

[8] R. Bechmann, A. Ballato, and T.J. Lukaszek, "Higher Order Temperature Coefficients of the Elastic Stiffnesses and Compliances of Alpha-Quartz," Proceedings IRE, Vol 50, pp. 1812-1822, Aug. 1962, p. 2451, Dec. 1962

[9] Landolt-Börnstein, Numerical data and functional relationships in science and technology, Group III, Crystal and solid state physics, Vol. 11, K.H. Hellwege and A.M. Hellwege Eds., Springer-Verlag Berlin Heidelberg - New York, 1979.

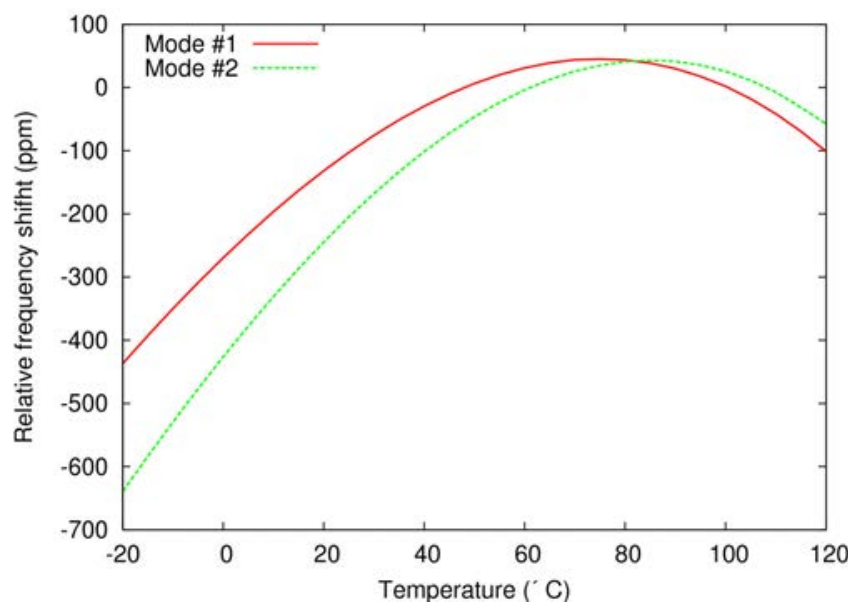

Figure 8. Thermal sensitivity of Love-wave under the grating of fig.6 for the two different modes emphasized in fig.7.
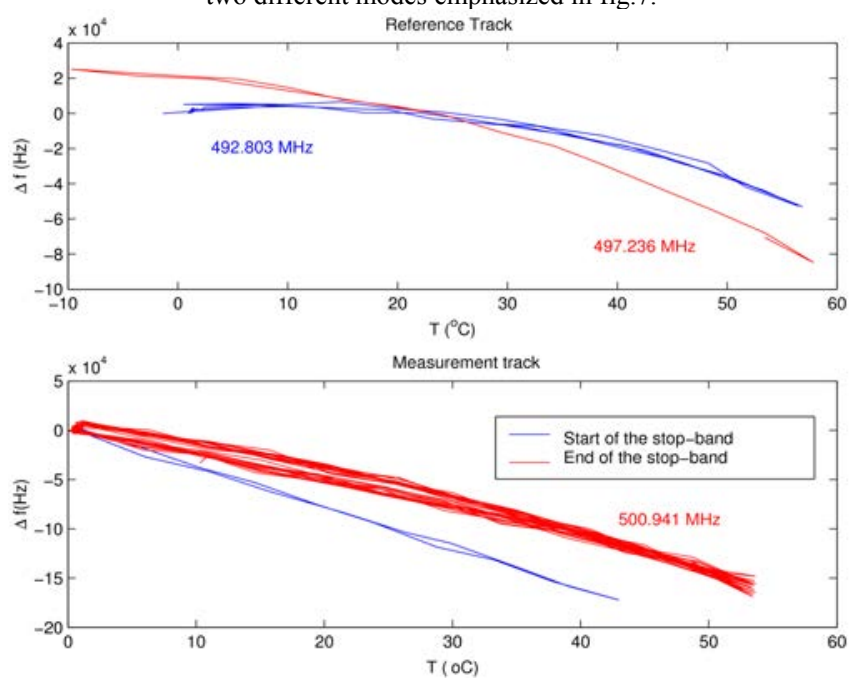

Figure 9. Experimental temperature drift of the beginning and end-of-thestopband frequencies of passivated Love wave resonators. 